\title{
Paediatric imaging in South Africa - From baby steps to giant strides
}

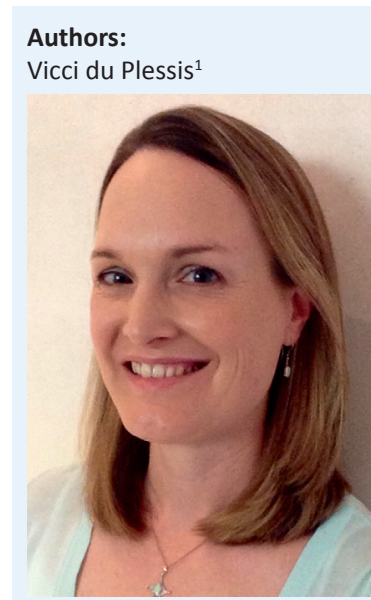

Farhana Suleman ${ }^{2}$

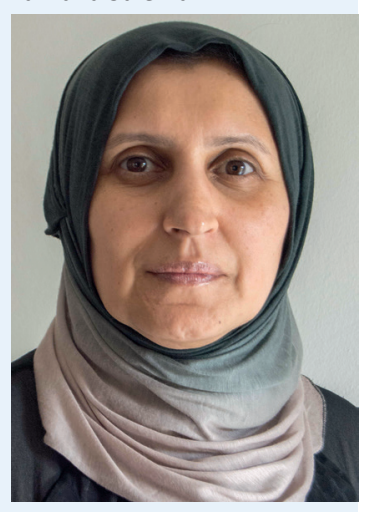

Affiliations:

${ }^{1}$ South African Society of Paediatric Imaging (SASPI), Cresta, South Africa

${ }^{2}$ Department of Radiology, Grey's Hospital, South Africa

Correspondence to: Vicci du Plessis

Email:

paediatric.imaging.sa@ gmail.com

Postal address:

Private Bag X9001,

Pietermaritzburg 3200,

South Africa
Read online:

Scan this QR code with your smart phone or mobile device to read online.
The South African Society of Paediatric Imaging (SASPI) is honoured to guest edit this special issue of the SA Journal of Radiology $(S A J R)$ in celebration of the International Day of Radiology (IDoR) on 08 November, which is dedicated to paediatrics this year.

Formally inaugurated in March 2011, SASPI is a sub-group of the Radiological Society of South Africa (RSSA), consisting of over 100 members including registrars, radiologists, paediatric radiology fellows, and paediatric radiologists. SASPI is expanding globally, having spearheaded the foundation of the African Society of Paediatric Imaging (AfSPI), a sub-group of the African Society of Radiology (ASR). SASPI is also strongly affiliated to the World Federation of Pediatric Imaging (WFPI). Founding and past SASPI Chair and AfSPI President Savvas Andronikou established the WFPI Outreach Programme, and the present SASPI Chair and AfSPI President Jaishree Naidoo currently represents South Africa at international conferences as WFPI vice-Secretary.

Over the past five years, SASPI has striven for excellence in paediatric imaging in South Africa and beyond. Supported by our few, but world-renowned, South African paediatric radiologists (all of whom have published articles in the current special issue), SASPI has pursued the following aims:

\section{To optimise national paediatric imaging protocols}

The SASPI/RSSA paediatric imaging guidelines (based on the Red Cross War Memorial Children's Hospital guidelines) were compiled during 2012 and have since been available to all South African radiologists on the RSSA website. SASPI thanks Savvas Andronikou, Jaishree Naidoo, Tracy Kilborn, Richard Pitcher, Nicky Wieselthaler and Aadil Ahmed for contributing to this initiative.

\section{To promote the reduction of radiation exposure to children during imaging}

This has been a major goal for SASPI, as reflected in the national guidelines, as well as the personal pledge by each of our members to support this cause in accordance with the 'Image Gently' campaign. This priority for South African paediatric imaging has also filtered its way into written questions for the FCRadDiag(SA) exit examination. It is therefore appropriate and our privilege to have an article in this special issue by Kimberly Applegate, one of the steering committee members of the Alliance for Radiation Safety in Pediatric Imaging.

\section{To advance education in paediatric radiology}

The Red Cross War Memorial Children's Hospital Paediatric Radiology Fellowship has produced five fellows since 2009, thanks to its fine teachers: Tracy Kilborn, Nicky Wieselthaler, Richard Pitcher and Ebrahim Banderker. Initially it was funded by the Harry Crossley Foundation, and is currently possible thanks to the generous financial support of Drs Schnetler, Corbett and Partners Inc., Diagnostic Radiologists. The Charlotte Maxeke Johannesburg Academic Hospital Observership has been kindly facilitated by Jaishree Naidoo, who will also be co-ordinating the upcoming Nelson Mandela Children's Hospital Fellowship.

Few will forget the first SASPI/RSSA Paediatric Imaging Congress in Sandton in November 2012, a phenomenal success and a milestone for South African paediatric radiology. 2016 marks the year of the 2nd SASPI/RSSA Paediatric Imaging Congress to be held at Spier Estate, Stellenbosch, from 04-06 November. The esteemed panel of local and international speakers will focus on abdominal (gastro-intestinal and genito-urinary) and chest (pulmonary and cardiac) imaging, with a strong emphasis on radiation protection of paediatric patients.

How to cite this article: Du Plessis V, Suleman F. Paediatric imaging in South Africa - From baby steps to giant strides. S Afr J Rad. 2015;19(2); Art. \#951, 2 page. http://dx.doi.org/10.4102/sajr.v19i2.951

Copyright: @ 2015. The Authors. Licensee: AOSIS OpenJournals. This work is licensed under the Creative Commons Attribution License. 
As an additional IDoR 2015 initiative, SASPI initiated a series of educational paediatric imaging webinars in collaboration with the RSSA and the Children's Hospital of Philadelphia (CHOP). These were very well-supported and received outstanding feedback from attending delegates. Thank you to Tanyia Pillay, Kassa Darge and the RSSA and CHOP administrative staff.

\section{To support outreach projects locally, regionally and internationally}

Apart from site visits to Durban, Pietermaritzburg, Bloemfontein and Ga-Rankuwa, SASPI has also organised outreach projects in Swaziland and Malawi. SASPI members have volunteered for local and international tele-reporting projects and also contribute to the WFPI's 'TB corner' via the WFPI outreach programme.

\section{To encourage paediatric radiology research}

By granting SASPI the unique opportunity to guest edit this issue, the $S A J R$ has assisted in achieving this final goal. SASPI would like to take this opportunity to thank the RSSA, the $S A J R$ editorial board (Razaan Davis and Farhana Suleman), the AOSIS publishing team, and of course all local and international contributors (authors and reviewers) for their tremendous support in pioneering this remarkable special paediatric issue.

Yours in paediatric imaging

Vicci du Plessis

Secretary: South African Society of Paediatric Imaging (2011 - 2015)

Guest editor: SAJR Paediatric Special Issue

\section{A giant leap forward for the SAJR}

A huge advantage of online publication of journals, especially imaging journals, is the improved content that can be afforded to the readership in terms of quality and diversity of the images provided. If a picture says a thousand words, then a video can tell an entire story, and this special paediatric issue of the $S A J R$ introduces for the first time an article with video content. When authors from the University of Pretoria approached the editorial team to discuss the feasibility of including videos in the article 'Heterotaxy syndrome: This is the left, right?', it generated huge excitement but, as always with publications, cost became a huge factor. Refusing to be discouraged, the authors then approached the Department for Education Innovation at the Faculty of Health Sciences, University of Pretoria, who willingly provided assistance in preparing the videos, uploading them onto the website, and providing the URL links for the article at no cost to the authors or publishers. The result is a major step forward for the journal in keeping with international publication trends, and confirms the commitment of the editorial team, and in particular the editor-in-chief Razaan Davis, to establishing the $S A J R$ as a proudly South African journal.

We take this opportunity to thank Marizanne Booyens, Anton van Dyk and Hannelie Untiedt of the Department for Education Innovation at the University of Pretoria for their assistance with the images and videos that enables us to take the $S A J R$ into a new era.

Farhana Suleman

South African Journal of Radiology 